\title{
"Society of Hematologic Oncology (SOHO) State of the Art Updates and Next Questions"- Treatment of ALL
}

\author{
Sabina Chiaretti, ${ }^{1}$ Elias Jabbour, ${ }^{2}$ Dieter Hoelzer ${ }^{3}$
}

The outcome of adult acute lymphoblastic leukemia (ALL) has substantially improved by adopting pediatric-inspired regimens, and approximately half of the patients are nowadays cured. The evaluation of minimal residual disease currently represents the most important prognostic indicator, which drives treatment algorithms, which include allogeneic stem cell transplantation (allo-SCT) allocation. Indeed, for high-risk patients, allo-SCT should be pursued as soon as possible, whereas in standard-risk patients this procedure should be avoided also in light of related toxicity and because there are no significant benefits. Furthermore, better characterization of the molecular genetic events can drive therapeutic decisions: a historical example in this respect is represented by the use of tyrosine kinase inhibitors (TKIs) in Philadelphia chromosome-positive ALL; in the upcoming future, TKls might be used also in other subgroups, such as breakpoint cluster region/Abelson 1-like cases and others with deregulated tyrosine kinases. Finally, the greatest progress is currently achieved with new immunotherapies targeting frequently expressed surface antigens in ALL. It is also a new chance for elderly ALL patients, so far spared from intensive chemotherapy and allo-SCT. These targeted therapies will substantially change this treatment algorithm and the great challenge is to find optimal sequence of the extended therapy options in an individual patient.

Clinical Lymphoma, Myeloma \& Leukemia, Vol. 18, No. 5, 301-10 @ 2018 Elsevier Inc. All rights reserved.

Keywords: Acute lymphoblastic leukemia, Adults, Chemotherapy, Immunotherapy, Molecular targets and molecular subgroups

\section{Introduction}

In children, the cure rate is now $\geq 80 \%$. Despite using the same drugs, in adults the cure rate is lower, because of a higher risk profile, chemoresistance, and increased toxicity. In 10 published studies up to 2013 the cure rate was $35 \%$. This changed in the recent years: a cure rate of $50 \%$ to $60 \%$, even higher in certain subgroups, is obtained. Reasons for improvement are better risk stratification, comprising conventional and genetic classification factors and evaluation of minimal residual disease (MRD). Allogeneic (allo-) stem cell transplantation (SCT) contributed. In Philadelphia chromosome-positive $\left(\mathrm{Ph}^{+}\right)$acute lymphoblastic leukemia

${ }^{1}$ Hematology, Department of Cellular Biotechnologies and Hematology, "Sapienza" University, Rome, Italy

${ }^{2}$ Department of Leukemia, University of Texas M.D. Anderson Cancer Center, Houston, TX

${ }^{3}$ Onkologikum, Frankfurt am Museumsufer, Frankfurt, Germany

Submitted: Mar 27, 2018; Accepted: Mar 28, 2018; Epub: Apr 3, 2018

Address for correspondence: Dieter Hoelzer, MD, PhD, University of Frankfurt, Germany, Onkologikum, Frankfurt am Museumsufer, Gartenstr., 134 D-60596 Frankfurt, Germany

E-mail contact: hoelzer@em.uni-frankfurt.de
(ALL) - the poorest prognostic subtype - the application of tyrosine kinase inhibitors (TKIs) has improved the survival to $60 \%$. Nowadays, patients remaining $\mathrm{MRD}^{+}$after induction therapy have the worse outcome.

Immunotherapeutic approaches have been successfully explored in ALL. B-lineage ALL blasts express surface antigens effectively targeted by monoclonal antibodies: rituximab for CD20, inotuzumab ozogamicin for CD22, and blinatumomab for CD19. These immunotherapies improved outcome in relapsed/refractory (R/R) and $\mathrm{MRD}^{+}$ALL patients and are currently explored in first-line disease. A new option is represented by chimeric antigen receptor (CAR), targeting CD19; novel CAR generations, targeting CD22, or CD19 and CD22, are under way.

They will substantially change treatment algorithms, implying reduction of chemotherapy-intensity, thus permitting management also of elderly patients, and reduced allocation to allo-SCT. This review will focus on the achievement and integration of these targeted therapies and how the treatment paradigms for ALL will change.

\section{Diagnosis}

The diagnosis of ALL relies on different steps. 


\section{Treatment of ALL}

Cytomorphology. Cytomorphology must be evaluated for differential diagnosis with acute myeloid leukemia (AML). The FrenchAmerican-British (FAB) classification previously distinguished 3 FAB subtypes: FAB1 and FAB2 are not used anymore whereas a FAB3 is almost invariably associated with a mature B-lineage ALL.

Flow Cytometry. Immunophenotyping using multichannel flow cytometry (MFC) is crucial for ALL diagnosis, detection, and monitoring of MRD, and potential targeted therapy. Recently, novel MFC techniques have been developed by the EuroFlow consortium to ensure accurate methodologies and to increase sensitivity. ${ }^{1,2}$

B-Lineage ALL. Of the different types of ALL $75 \%$ to $80 \%$ are of $\mathrm{B}-$ cell lineage. The markers for B-lineage diagnosis are CD19, CD20, $\mathrm{CD} 22, \mathrm{CD} 24$, and CD79a. Four differentiation stages are recognized (see Table 1, B-lineage ALL). In 38\% of patients, an aberrant coexpression of myeloid markers is detected. ${ }^{3}$ Although there is no striking correlation between immunophenotype and well established cytogenetic subsets, a pro-B stage is often associated with $\mathrm{t}(4 ; 11) / \mathrm{mixed}-$ lineage-leukemia (MLL) (alias lysine methyltransferase $2 \mathrm{~A}$ ) rearrangements (MLL-r), and a pre-B with $\mathrm{t}(1 ; 19) /$ transcription factor 3 (TCF3)-PBX homeobox 1 (PBX1) rearrangements. ${ }^{4}$ Neuron-glial antigen 2 (NG2) is detected in $\mathrm{t}(4 ; 11)^{+}$cases $^{5}$ and CD66c has been associated with $\mathrm{Ph}^{+}$ALL. ${ }^{6}$

T-Lineage ALL. T-lineage ALL represents $20 \%$ to $25 \%$ of ALL. Crucial markers are CD1a, CD2, CD3 (surface as well as cytoplasm), CD4, CD5, CD7, and CD8: its diagnosis relies on CD3 surface/cytoplasmic expression. CD34 and myeloid antigens CD13 and/or CD33 can be expressed (34\% and $24 \%$, respectively). ${ }^{3}$ According to the stage of differentiation, 4 T-ALL subtypes can be identified (Table 1, T-lineage ALL). The so-called early T-precursor (ETP)-ALL was included in the most recent World Health Organization classification ${ }^{7}$ : it lacks CD1a and CD8 expression, has weak CD5 expression, and at least 1 myeloid and/or stem cell marker and is discussed separately. ${ }^{8}$

Conventional Cytogenetics. In B-lineage ALL, several lesions with prognostic significance were identified using conventional karyotyping.
Good prognosis aberrations include $\mathrm{t}(12 ; 21)$ /ETS variant 6 (ETV6)-Runt related transcription factor 1 (RUNX1) and high hyperdiploidy (51-65 chromosomes).

Aberrations associated with an intermediate risk comprise the normal diploid subset, isolated trisomy 21 , trisomy 8 , del(6q), and $\mathrm{t}(1 ; 19) / \mathrm{TCF} 3-\mathrm{PBX} 1$ : in the latter, the dismal outcome is overcome by the current therapeutic approaches.' Furthermore, intrachromosomal amplification of chromoosme 21, defined as a consistent amplification of the RUNX1 locus deriving from a massive chromothripsis and identified in $2 \%$ of cases, was regarded as a poor prognostic aberration: its prognostic effect might vary according to treatment intensity. ${ }^{10}$ Translocations involving the immunoglobulin heavy locus (IGH) locus recognize different fusion partners, the most frequent being cytokine receptor like factor 2 (CRLF2), and CCAAT/enhancer binding protein genes and inhibitor of DNA binding 4, HLH protein. ${ }^{11}$

Poor prognostic aberrations are represented by MLL-r, monosomy 7, hypodiploidy/low hypodiploidy (and the related near-triploid), and t(17;19)/TCF3-HLF, PAR BZIP transcription factor. ${ }^{12}$ Patients with $\mathrm{t}(9 ; 22)$ /breakpoint cluster region (BCR)Abelson (ABL1) rearrangements/fluorescent in situ hybridizationamplified $\left(\mathrm{Ph}^{+}\right)$were considered the worse subgroup, but this does not hold true in the TKI era. ${ }^{13}$

In T-lineage ALL, ${ }^{14}$ cytogenetic aberrations involve $14 \mathrm{q} 11$ breakpoints $(\mathrm{t}(10 ; 14), \mathrm{t}(11 ; 14), \mathrm{t}(1 ; 14)$, etc). The $\mathrm{t}(8 ; 14)$, involving $\mathrm{q} 24 ; \mathrm{q} 11$, is associated with an aggressive presentation. ${ }^{15}$

Copy Number Aberrations. Genome-wide technologies identified novel lesions. ${ }^{16}$ The most frequent is the Ikaros (IKZF1) deletion $(\triangle I K Z F 1),{ }^{17,18}$ identified in approximately $80 \%$ of cases with $\mathrm{Ph}^{+}$ ALL and in approximately $30 \%$ of B-lineage ALL lacking $\mathrm{Ph}$ chromosome, in children as well as adults, with a higher incidence in adults. Whereas $\triangle I K Z F 1$ have prognostic significance in $\mathrm{Ph}^{-}$ ALL pediatric cases, their role is more controversial in adult ALL: it is emerging that their effect is limited to cases with additional genomic lesions, particularly cyclin dependent kinase inhibitor $2 \mathrm{~A}$ and $2 \mathrm{~B}(C D K N 2 A / B)$ and paired box 5 .

$C D K N 2 A / B$ deletions are identified in $40 \%$ of cases, and negatively influence outcome in $\mathrm{Ph}^{+}$as well as $\mathrm{Ph}^{-} \mathrm{ALL}$; similar results were reported for early $\mathrm{B}$ cell factor $1(E B F 1)$ deletions, detected in

Table 1 Immunophenotypic Characterization of B-Lineage ALL

\begin{tabular}{|c|c|c|c|c|c|c|c|c|}
\hline B-Lineage ALL & TdT & CD19 & CD79 & cCD22 & CD10 & $\operatorname{clg} \mu$ & $\operatorname{slg} \mu$ & sk/ $/ \lambda$ \\
\hline Pro-B & + & + & + & + & - & - & - & - \\
\hline Common & + & + & + & + & + & - & - & - \\
\hline Pre-B & + & + & + & + & $+1-$ & + & - & - \\
\hline B-mature & - & + & + & + & - & + & + & + \\
\hline T-Lineage ALL & TdT & cCD3 & CD7 & CD2 & CD5 & CD1a & sCD3 & $\gamma / \delta$ or $\alpha / \beta$ \\
\hline Pro-T & + & + & + & - & - & - & - & - \\
\hline Pre-T & + & + & + & + & + & - & - & - \\
\hline T-cortical & + & + & + & + & + & + & $+/-$ & - \\
\hline T-mature & $+1-$ & + & + & + & + & - & + & $+/-$ \\
\hline
\end{tabular}

Abbreviations: $\mathrm{ALL}=$ acute lymphoblastic leukemia; $\mathrm{CCD}=$ cytosplasmic $\mathrm{CD} ; \mathrm{clG} \mu=$ cytoplasmic $\mathrm{IG} \mu$; $\mathrm{sCD}=\mathrm{surface} \mathrm{CD}$; slg $\mu=$ surface $\mathrm{IG} \mu$; sk $\lambda=\operatorname{surface}$ $\mathrm{k} \lambda$; TdT $=$ terminaldeoxytrasnsferase. 
approximately $10 \%$ of adult patients, and RB transcriptional corepressor 1 deletions. ${ }^{19,20}$

Gene Mutations and Rearrangements in B-Lineage and T-ALL. Regarding genome-wide sequencing identified novel mutations and rearrangements: the most frequent involve the RAS pathway (KRAS proto-oncogene, GTPase/NRAS proto-oncogene, GTPase $[N / K$ $R A S]$, Fms related tyrosine kinase 3 (FLT3), protein tyrosine phosphatase, non-receptor type 11 (PTPN11), neurofibromin 1 $(N F 1)$, and B-raf proto-oncogene, serine/threonine kinase mutations), detected in $>40 \%$, prevailing in the hyperdiploid and MLL-r cases and increasing at relapse. ${ }^{21}$

More rare mutations affect the Janus kinase (JAK)/signal transducer and activator of transcription (STAT) pathway (ie, JAK1/2, $J A K 3$ [in T-ALL], interleukin 7 receptor $[I L 7 R]$, rarely $C R L F 2$ mutations, $\mathrm{SH} 2 \mathrm{~B}$ adaptor protein 3 and interleukin 2 receptor subunit beta. JAK/STAT mutations are frequent in BCR/ABL1-like ALL and cases with IGH translocations. ${ }^{21}$

The RAS as well as the JAK/STAT pathway mutations are detected in B- and T-lineage ALL.

Fusion genes involving tyrosine kinases are found, mostly in the BCR/ABL1-like ALL cases; other novel fusion genes include $M E F 2 D$, double homeobox, 4, ERG, ETS transcription factor, and CRLF2. ${ }^{22,23}$

In T-ALL, Notch1/F-box and WD repeat domain containing 7 lesions represent a frequent event (approximately 60\%); JAK/STAT and RAS pathway mutations can be detected, with a variable frequency depending on the cohorts (10\%-30\%). Their recognition is important because they have prognostic and therapeutic implications. ${ }^{24}$

\section{Prognostic Factors and Risk Stratification}

Identification of prognostic parameters at diagnosis-including age, white blood cell count, specific immunophenotypes and cytogenetic/genetic aberrations_allow patients' stratification into risk groups: standard-risk (SR) patients, with a good chance of cure

\section{Table 2 Prognostic Risk Factors}

\begin{tabular}{l|c}
\hline $\begin{array}{l}\text { Risk Factor } \\
\text { Patient-Related }\end{array}$ & Risk Subset \\
\hline Age & $40 / 55 / 65$ \\
\hline Performance status & $>1$ \\
\hline $\begin{array}{l}\text { Disease-Related } \\
\text { WBC }\end{array}$ & $>30,000 \times 10^{9} / \mathrm{L}$ in B-lineage ALL \\
& $>100,000 \times 10^{9} / \mathrm{L}$ in T-lineage ALL \\
\hline Phenotype & Pro-B/Pro-T/ETP/mature T-ALL \\
\hline Cytogenetics & $B C R-A B L 1^{+} / M L L^{+} / B C R-A B L 1^{+}$ \\
\hline Genetics & $B C R / A B L 1$-like, CDKN2A/B, EBF1 \\
\hline Response to Therapy & Poor prednisone response \\
\hline $\begin{array}{l}\text { Corticosteroid sensitivity } \\
\text { (blast count after pre-phase) }\end{array}$ & $>1 \times 10^{9} / \mathrm{L}$ ) \\
\hline $\begin{array}{l}\text { Early blast cell response (BM } \\
\text { morphology) }\end{array}$ & $>1$ day 15 \\
\hline Time to CR (number of courses) & MRD positivity (postinduction) \\
\hline MRD & \\
\hline
\end{tabular}

Abbreviations: $\mathrm{ALL}=$ acute lymphoblastic leukemia; ETP = early T-precursor; $\mathrm{BM}=$ bone marrow; $\mathrm{MRD}=$ minimal residual disease; $\mathrm{WBC}=$ white blood cell. using chemotherapy, and high-risk (HR) patients with one or more risk factors. HR patients are most often candidates for allo-SCT in first complete remission (CR; Table 2). ${ }^{25}$ Currently, MRD is regarded as the most important prognostic factor.

\section{Minimal Residual Disease Assessment, Terminology, and Effect on Outcome}

Minimal residual disease is the detection, using flow cytometry (FCM) or molecular analysis, of residual leukemic cells. MRD evaluation must be performed at the end of induction for treatment intensification and during consolidation because MRD persistence/ reappearance $\left(>10^{-4}\right)$ can drive therapeutic decisions.

Minimal residual disease techniques must be sensitive $\left(\leq 10^{-4}\right)$, applicable, reliable, and affordable: the most common are FCM, polymerase chain reaction (PCR) analysis of rearranged immunoglobulin and T-cell receptor (allele-specific oligonucleotide [ASO]PCR) genes, and real-time quantitative and polymerase chain reaction (RQ-RT-PCR) methods for fusion genes, if present. ${ }^{26}$ FCM is less sensitive than ASO-PCR and RQ-RT-PCR, mostly when 4- and 6-color are used; ASO-PCR represents the most reliable approach, but it is time-consuming; finally, RQ-RT-PCR is highly sensitive $\left(10^{-4}-10^{-6}\right)$ and easy to perform; however, full standardization of all steps and international quality assurance systems are not yet available. Intensive research is ongoing to validate novel tools, such as nextgeneration sequencing ${ }^{27}$ and digital droplet PCR. ${ }^{28}$

Minimal residual disease terminology is summarized in Table $3 .^{29}$

Achievement of molecular complete molecular remission (CMR) represents currently the most relevant independent prognostic factor for disease-free survival (DFS) and overall survival (OS), as shown in a meta-analysis of $>13,000$ patients (children and adults) with ALL. ${ }^{30}$ Patients in CMR after induction had significantly superior outcome, with a DFS of $54 \%$ to $74 \%$, compared with $17 \%$ to $40 \%$ for MRD-positive patients. Patients with molecular failure after induction should proceed to a targeted therapy to reduce the tumor load followed by an allo-SCT. ${ }^{31}$

Will MRD Evaluation Replace Pretherapeutic Risk Factors?. The question is whether MRD evaluation overcomes the diagnostic risk factors or they should be integrated. A practical approach is to enter the conventional prognostic factors and MRD into a decision algorithm. Thereby, SR patients in CMR (approximately 90\%-95\%), will remain as such, whereas those who are $\mathrm{MRD}^{+}$will be shifted to HR. Clinically defined HR patients are potential candidates for alloSCT in first CR (CR1): it is not clear how to proceed if they achieve a CMR, because some studies suggest no benefit from allo-SCT.

If $\mathrm{MRD}$ is not available, stratification should rely on diagnostic clinical factors.

Unfortunately, $20 \%$ to $30 \%$ of adult ALL patients who are $\mathrm{MRD}^{-}$after induction will relapse. Reasons include loss of sensitivity, clonal/subclonal evolution, extramedullary relapse, different CD19 escape mechanisms, and others. ${ }^{29}$

\section{Treatment Principles}

Pre-Phase Therapy. In ALL, treatment should start immediately, with a pre-phase-which represents the timing for diagnostic workup-consisting of corticosteroids, sometimes in combination with vincristine or cyclophosphamide. 


\section{Treatment of ALL}

Table 3 Response Parameters According to MRD

\begin{tabular}{|c|c|}
\hline Terminology & Definition \\
\hline Complete (Hematologic) Remission & Leukemic cells not detectable using light microscopy ( $<5 \%$ blast cells in bone marrow) \\
\hline Complete Molecular Remission, MRD-Negativity & Patient in complete remission, MRD not detectable, $\leq 0.01 \%=\leq 1$ leukaemia blast cell in 10,000 \\
\hline Molecular Failure/MRD-Positivity & Patient in complete hematologic remission but not in molecular complete remission $>0.01 \%$ \\
\hline Molecular Relapse/MRD-Positivity & $\begin{array}{c}\text { Patient still in complete remission, had previous molecular complete remission; Leukemic blast cells in bone } \\
\text { marrow not detectable }(<5 \%)\end{array}$ \\
\hline Hematologic Relapse & $>5 \%$ ALL cells in bone marrow/blood \\
\hline
\end{tabular}

Abbreviations: $\mathrm{ALL}=$ acute lymphoblastic leukemia; $\mathrm{MRD}=$ minimal residual disease.

Intrathecal central nervous system (CNS) prophylaxis should be carried out as soon as possible.

Remission Induction. The goal of induction therapy is achievement of a CR and/or CMR, usually within 2 induction cycles (approximately 16-22 weeks). Most regimens are centered on vincristine, corticosteroids, and anthracycline (daunorubicin, doxorubicin, idarubicin), with or without cyclophosphamide or cytarabine. L-asparaginase is the only ALL-specific drug that depletes asparagine levels and was mainly explored in pediatric trials; it is currently used also in adults. Pegylated asparaginase significantly increases the period of asparagine depletion. Dexamethasone is often preferred to prednisone, because it penetrates the blood-brain barrier (BBB) and also acts on resting leukemic blast cells.

Two regimens are mostly used in adult ALL: one-inspired by the pediatric Berlin-Frankfurt-Münster protocols_-is often applied in European adult ALL trials; the other is the hyper-CVAD (cyclophosphamide, vincristine, doxorubicin, and dexamethasone), with 2 different alternating intensive chemotherapy cycles, identical for induction and consolidation, for a total of 8 cycles. ${ }^{32}$ It is used in the United States, but also in other parts of the world.

Consolidation Therapy. The rationale to use systemic high-dose (HD) therapy is to achieve a CMR and reach sanctuary sites (CNS and testicles). Most protocols use 6 to 8 courses with HD methotrexate (MTX) or HD cytarabine with or without asparaginase.

Maintenance Therapy: Still a Backbone of ALL. Maintenance therapy consists of daily 6-mercaptopurine and weekly MTX. In some regimens, reinductions are given: in one randomized study, the maintenance arm with reinforcement cycles was not superior to conventional maintenance ( $37 \%$ vs. $38 \%$ at 8 years). ${ }^{33}$ Maintenance duration of 2.5 to 3 years is recommended; its omission worsens outcome in B-lineage ALL, but not in T-ALL, ${ }^{34}$ and is not required in mature B-ALL. ${ }^{35}$

Age-Adapted Protocols. The outcome of ALL is strictly related to age, with cure rates of $80 \%$ to $90 \%$ in children, and $30 \%$ in elderly/ frail ALL patients (Table 4). Therefore, age-adapted protocols have emerged, dictated by toxicities and comorbidities. Although there is no uniform consensus, the following age groups are considered:

- Adolescents and young adults (AYA), defined from 15 to 18 and 35 to 40 years, respectively;

- Adults, age range 35 to 40 and up to years and younger to 60 years;

- Elderly patients older than 55 to 60years; and

- Frail patients not suitable for any intensive therapy, older than 70 or 75 years.

Adolescents and Young Adults. Pediatric-inspired therapy provides increased drug intensity, including larger cumulative doses of corticosteroids, vincristine, L-asparaginase, and consequent CNS-directed therapy, with a reduced role of allo-SCT. In a meta-analysis including 11 trials and 2489 AYA patients, pediatric-inspired regimens were superior to conventional adult chemotherapy. ${ }^{36}$ In recent studies for AYAs, ${ }^{37-39}$ survival rates at 5 years were $67 \%$ to $78 \%$, compared with $34 \%$ to $41 \%$ with former protocols.

Adults. In 20 studies from 1998 to 2016, including nearly 8000 adults, the weighted mean for CR rate was $84 \%$ (94\%-93\%) and the 5 -year OS approximately $35 \%$. Using current approaches, the CR rate increased to $80 \%$ to $90 \%$, being higher for SR patients $(\geq 90 \%)$ and lower in HR patients (70\%-80\%). A CMR rate $\geq 70 \%$

Table 4 Outcome of Adult ALL According to Age

\begin{tabular}{l|c|c|c|c|c|c|c}
$\begin{array}{l}\text { Type of } \\
\text { Treatment }\end{array}$ & Time Period & Studies, n & Patients, $\mathbf{n}$ & $\begin{array}{c}\text { Age (Range), } \\
\text { Years }\end{array}$ & $\begin{array}{c}\text { CR Rate } \\
\text { (Range), \% }\end{array}$ & $\begin{array}{c}\text { Early Death } \\
\text { (Range), \% }\end{array}$ & $\begin{array}{c}\text { Overall Survival } \\
\text { (Range), \% }\end{array}$ \\
$\begin{array}{l}\text { Pediatric-Inspired } \\
\text { for AYAs }\end{array}$ & $2008-2015$ & 6 & 832 & $27(15-60)$ & $93(85-98)$ & $5(1-7)$ & $70(60-78)$ \\
\hline $\begin{array}{l}\text { Adult Trials } \\
\begin{array}{l}\text { Elderly Age- } \\
\text { Specific Protocols }\end{array}\end{array}$ & $1998-2016$ & 20 & 7961 & $32,7(12-92)$ & $84(74-93)$ & $7(1-10)$ & $36(27-60)$ \\
\hline
\end{tabular}

Abbreviations: $\mathrm{ALL}=$ acute lymphoblastic leukemia; $\mathrm{AYA}=$ adolescents and young adults aeighted mean. 
can be achieved in SR patients and approximately 50\% in HR patients. $^{31}$

The OS for SR patients is $50 \%$ to $70 \%$ with chemotherapy alone. The outcome for HR patients has also increased to approximately $50 \%$ when allo-SCT in CR1 is given. Prospective adult studies applying the same drugs and time dose-intensity, without the term "pediatric-inspired," achieved identical results compared with AYAs, with survival rates of $60 \%$ to $70 \%$ or more. ${ }^{40-43}$

Elderly Patients. The incidence of ALL increases after the fifth decade. ${ }^{44}$ Different approaches have been applied. ${ }^{45,46}$ So far, neither a palliative treatment with CR rates of $43 \%(34 \%-53 \%)$, an early death rate of $24 \%(18 \%-42 \%)$, and an OS of only 7 months (3-10 months), nor an intensive chemotherapy designed for adult ALL with CR rates of $56 \%(40 \%-81 \%)$, early death rate of $23 \%(6 \%-42 \%)$, and an OS of 14 months (3-29 months) is optimal. The current general principle is a less intensive induction based on corticosteroids, vincristine and asparaginase, avoiding anthracyclines and alkylating agents, to reduce early treatmentrelated death;upon induction, reduced intensive consolidation cycles with MTX and citosine-arabinoside (ARA-C) are given. In 9 recent prospective studies for older patients (55-81 years), with this scheme, the CR rate was $71 \%(43 \%-90 \%)$, early death decreased to $15 \%(0-36 \%)$, and OS increased to 42 months.

Treatment Approaches With Reduced Intensity. Reasons to reduce chemotherapy intensity are the difficulty in administering intensive regimens and allo-SCT in patients older than 50 years and the rate of toxic deaths.

Low-intensity chemotherapy is of interest when combined with targeted therapies (eg, with inotuzumab ${ }^{47}$ ), that lead to a CR rate of $98 \%$ and a 2 -year progression-free survival (PFS) of $52 \%$, or with dasatinib. ${ }^{48}$

\section{Acute Lymphoblastic Leukemia Subtypes}

Philadelphia Chromosome-Positive ALL. Patients with $\mathrm{Ph}^{+}$ALL constitute approximately $25 \%$ of adult B-ALL, with an increasing incidence to approximately $50 \%$ among elderly patients. In the preimatinib era, $\mathrm{Ph}^{+} \mathrm{ALL}$ was the poorest ALL subtype. The $\mathrm{CR}$ rates were $60 \%$ to $70 \%$, CMR rates $<5 \%$, and OS with chemotherapy alone $10 \%$ and approximately $30 \%$ with allo-SCT.

The results improved substantially when the first-generation TKI imatinib became available. CR rates increased to $80 \%$ to $90 \%$, CMR rate to $\geq 50 \%$, and the 5 -year survival to $\geq 50 \%$. ${ }^{13}$

In $\mathrm{Ph}^{+} \mathrm{ALL}$, a chemotherapy-free induction regimen, consisting only of prednisone combined with the TKI dasatinib was applied in prospective trials. This therapy avoids induction deaths, resulting in a CR rate of nearly $100 \%$ and can be given without age limit, ${ }^{49}$ avoiding allo-SCT in CR1, if feasible. These patients have to be carefully monitored and if a molecular relapse, mutation, or intolerability is emerging, therapy must be intensified. Also in the EWALL (European Working Group for Adult Acute Lymphoblastic Leukemia) studies, reduced intensive chemotherapy was given in combination with dasatinib ${ }^{48}$ or nilotinib. ${ }^{50}$ The CR rates were very high with $>90 \%$ because of a low rate of deaths in induction and remission.
Ponatinib has been tested in a phase II single-arm trial for previously untreated $\mathrm{Ph}^{+}$ALL patients in combination with hypercyclophosphamide, vincristine, doxorubicin and dexamethasone; initially, ponatinib was given at $45 \mathrm{mg}$ daily, but the study was amended after 2 fatal myocardial events. After this modification no further vascular events were reported. The CR, complete cytogenetic response (CCyR), major molecular response, and CMR rates were $98 \%, 98 \%, 97 \%$, and $77 \%$, respectively; no benefit in performing allo-SCT was observed. ${ }^{51}$ A phase II trial (Gruppo Italiano Malattie EMatologiche dell'Adulto [GIMEMA] 1811) with ponatinib (with steroids) in newly diagnosed elderly patients resulted in high rates of CR, CCyR, and CMR (90\%, 90\%, and 57\%, respectively), suggesting that ponatinib is effective also as monotherapy. ${ }^{52}$

Is Allo-SCT Essential for $\mathrm{Ph}^{+}$ALL?. Allogeneic stem cell transplantation was the only curative option for adults with $\mathrm{Ph}^{+}$ALL. With intensive chemotherapy induction, imatinib given throughout, and allo-SCT, the cure rate in younger patients was $50 \%$ to $60 \%$ in several trials. A prospective randomized trial indicates that allo-SCT is still associated with a better recurrence-free survival (RFS) in younger $\mathrm{Ph}^{+}$ALL patients. ${ }^{53}$ Currently, the tendency is to reduce/replace allo-SCT in CR1 by combining TKIs with low-dose chemotherapy or with immunotherapeutic approaches (see Immunotherapy in $\mathrm{Ph}^{ \pm} \mathrm{ALL}$ ).

The definition of a good-risk $\mathrm{Ph}^{+}$ALL patient is not established: therefore, the scientific effort should focus on the definition of patients who do unequivocally need transplantation. A potential algorithm for transplantation decision-making should include not only MRD, but also detailed genomic characterization at diagnosis.

Finally, autologous transplantation ${ }^{54}$ might be suggested in elderly or comorbid $\mathrm{Ph}^{+}$ALL patients who are repeatedly $\mathrm{MRD}^{-}$, and in countries where allo-SCT is limited for financial reasons.

Is Maintenance With a TKI After Allo-SCT Essentiallof Benefit? The gold standard is to give a TKI also after allo-SCT, as supported by a randomized study. ${ }^{55}$ However, toxicity is high for Imatinib And Dasatinib ${ }^{48}$ and higher for nilotinib ${ }^{56}$; finally, it is not clear for how long TKIs after allo-SCT should be given.

Is There an Optimal/Best TKI? The Issue of TKI Resistance. Faster and deeper molecular responses are achieved with second-generation TKIs, but it is open to what extent this transfers into a survival benefit.

One major issue is represented by the emergence of mutations, with $\mathrm{T} 315 \mathrm{I}^{48,57}$ inducing resistance to most TKIs, with the exception of ponatinib, which-possibly associated with chemotherapy_can be used as a bridge to allo-SCT; T315I-inclusive compound mutants confer resistance also to ponatinib. ${ }^{58}$ Therefore, for such cases, the use of alternative approaches is urgently required. Potential compounds include the vascular endothelial growth factor receptor inhibitor axitinib ${ }^{59}$ and ABL001 (asciminib). ${ }^{60}$

In general, mutational screening should be performed in patients with persistent MRD or progressive disease, and the recommendation is to switch to another TKI while screening for 


\section{Treatment of ALL}

TKI-resistant mutations and to adapt TKI choice according to the resistance profile.

Immunotherapy in $\mathrm{Ph}^{+}$ALL. A new option in $\mathrm{Ph}^{+}$ALL is immunotherapy.

Blinatumomab was evaluated in the phase II ALCANTARA trial in 45 patients with $\mathrm{R} / \mathrm{R} \mathrm{Ph}^{+}$ALL: 36\% achieved a response. The median RFS and OS were 6.7 and 7.1 months and 7 (44\%) of the responding patients received allo-SCT. ${ }^{61}$ Blinatumomab showed a high efficacy in patients with mutations and complex cytogenetic aberrations of blinatumomab. ${ }^{61,62}$

Blinatumomab was also given with ponatinib to 20 patients with $\mathrm{R} / \mathrm{R} \mathrm{Ph}^{+}$ALL or chronic myeloid leukemia (CML) in lymphoid blast phase with a response rate of $65 \%$. Median survival was 14 months. ${ }^{63}$

Blinatumomab is rapidly moving into the first-line setting, with a 3-arm randomized trial of the EWALL comparing imatinib versus ponatinib versus ponatinib together with blinatumomab in elderly $\mathrm{Ph}^{+}$ALL (reduced-intensity chemotherapy foreseen in all arms); the GIMEMA 2116, on the basis of an induction of dasatinib (with steroids) followed by a combination of dasatinib and blinatumomab, is currently enrolling participants.

Inotuzumab was given with bosutinib in $\mathrm{R} / \mathrm{R} \mathrm{Ph}^{+} \mathrm{ALL}(\mathrm{n}=14)$ and CML-lymohoid blast crisis $(\mathrm{n}=2)$, providing an objective response rate of $81 \%$ (CR 50\%) and median event-free survival (EFS) and OS of 8.8 months and 10.7 months, respectively. ${ }^{64}$

\section{BCR/ABL1-Like (Ph-Like) ALL}

BCR/ABL1-like (or Ph-like) ALL has been extensively characterized. These cases exert a transcriptional profile similar to that of true BCR/ABL1+ ALL, frequently harbor $\triangle I K Z F 1$ lesions, and CRLF2 deregulation, and can display a whole set of lesions that involve tyrosine kinases, the most frequent being of $\mathrm{ABL}$ class ( $A B L 1, A B L 2$, colony stimulating factor 1 receptor [CSF1R], platelet derived growth factor receptor alpha [PDGFRA], PDGFRB, in approximately $10 \%$ ), JAK/STAT (ie, JAK1, 2, and 3, $I L 7 R$, and CRLF2 mutations in $<10 \%)$, RAS pathway (N/K RAS, NF1, $P T P N 11$, and to a lesser extent FLT3), less frequently other uncommon tyrosine kinases, erythropoietin receptor gene and $M E F 2 D-C S F 1 R$ rearrangement. The type of lesion can vary from case to case, making their recognition difficult; some patients do not harbor any lesions. ${ }^{22,65,66}$

Clinically, $B C R / A B L 1$-like ALL displays a poorer outcome in terms of CR rate, MRD persistence, and long-term outcome.

At present, it is not clear if these cases should be treated differently upfront or, instead, therapy switch (or intensification with allo-SCT and targeted approaches) should be limited to cases remaining $\mathrm{MRD}^{+}$. Second, because of the plethora of genetic lesions, although there is a general consensus that patients should receive a TKI, the best TKI has not been defined. Two alternative approaches have been proposed: the first is on the basis of the underlying lesion, including dasatinib for cases with ABL class genes and JAK2 inhibitors, particularly ruxolitinib, for cases with JAK/STAT pathway lesions. This approach is not applicable in all hematologic centers; furthermore, preliminary results from MD Anderson Cancer Center on $9 \mathrm{R} /$ R BCR/ABL1-like patients did not show significant responses. ${ }^{67}$
Another approach could be the use of ponatinib, because in vitro experiments showed that it is able to reduce the proliferative rate in BCR-ABL1-like primary cells, regardless of the underlying molecular lesions. ${ }^{68}$ Third, the role of antibody constructs, namely blinatumomab and inotuzumab, remain to be definitively determined.

\section{Early T-Precursor ALL}

As mentioned, ETP-ALL was recognized by gene expression and can be easily recognized by FCM. ${ }^{8}$ ETP ALL occurs in children as well as in adults and represents approximately $10 \%$ of T-lineage ALL. Several genomic lesions have been identified, including mutations in DNA methyltransferase 3 alpha, FLT3, isocitrate dehydrogenase $(\mathrm{NADP}(+))$ 1, cytosolic, isocitrate dehydrogenase $(\mathrm{NADP}(+)) 2$, mitochondrial, and ETV6. Interestingly, FLT3 mutations can be detected in $\geq 35 \%$ of cases, thus implying the possibility of novel therapeutic strategies. ${ }^{69}$ Furthermore, mutations occurring in genes regulating cytokine receptors and RAS signaling (67\%), inactivating lesions disrupting hematopoietic development (58\%), and histonemodifying genes (48\%) have been reported, suggesting that ETPALL shares a similar genomic background with AML. Recent findings also highlight that ETP can be further stratified according to the levels of the homoebox A (HOXA) genes family, with the poor outcome confined to the $\mathrm{ETP}^{+} / \mathrm{HOXA}^{+}$subgroup. ${ }^{70}$ Clinically, this subgroup was initially associated with a dismal prognosis. The prompt recognition of ETP cases is improving their outcome: in fact, the use of intensified, pediatric-inspired and MRD-driven treatments has improved their outcome. Furthermore, the use of allo-SCT in first CR should be considered as the optimal choice: the Group for Research on Adult Acute Lymphoblastic Leukemia (GRAALL) group, in the context of GRAALL-2003 and -2005 studies, showed a noninferior outcome of ETP patients, mostly dependent on alloSCT allocation. ${ }^{71}$ Finally, the presence of AML-related features might prompt, in case of failures, to investigate the use of myeloiddirected therapies. Alternative strategies include the use of the BCL2, apoptosis regulator- inhibitor venetoclax, effective in patient-derived-xenografts. $^{72}$

\section{Allogenic Stem Cell Transplantation}

Allogenic stem cell transplantation was a curative approach in adult ALL, is still the best treatment option for patients in second or later $\mathrm{CR}$, and is highly recommended for $\mathrm{HR}$ patients in first $\mathrm{CR}{ }^{73,74}$ The outcome in $\mathrm{MRD}^{+}$patients after induction therapy can be improved by eradicating MRD levels: ongoing studies (eg, with blinatumomab) are extremely promising. ${ }^{75}$

Because allo-SCT is aggravated by treatment-related mortality (10\%-20\%) and impaired life quality because of chronic graft versus host disease, there are attempts to avoid allo-SCT in first CR.

Stem Cell Donors. Different donor sources for allo-SCT are available. There is increasing evidence that outcome is equivalent with siblings and compatible matched unrelated donors (MUD). Haploidentical-SCT (haplo-SCT) is being increasingly used, for cost reasons and prompt availability of a family donor. MUD versus haplo-SCT with an identical post-cyclophosphamide graft versus host disease prophylaxis in a European prospective randomized trials will be evaluated. 
Table 5 Blinatumomab Activity

\begin{tabular}{l|c|c|c|c}
\multirow{2}{*}{ Parameters } & Ph $^{+}$ALL & \multicolumn{2}{|c|}{ Ph $^{-}$R/R ALL } & Positive MRD \\
\cline { 2 - 5 } & Pivotal Phase II (ALCANTARA) & Confirmatory Phase II & Tower Phase III & BLAST Phase II \\
$\mathrm{n}$ & 45 & 189 & 271 & 116 \\
CR/CRh/CRi, \% & 36 & 43 & 45 & NA \\
MRD Negativity, \% & 88 & 82 & 76 & 78 \\
\hline OS, median (months) & 7.1 & 6.1 & 7.7 & 36 \\
\hline
\end{tabular}

Abbreviations: $\mathrm{ALL}=$ acute lymphoblastic leukemia; $\mathrm{CRh}=\mathrm{CR}$ with incomplete hematologic recovery; $\mathrm{CRi}=$ complete remission with incomplete recovery; $\mathrm{MRD}=$ minimal residual disease; $\mathrm{OS}=$ overall survival; $\mathrm{Ph}=$ Philadelphia chromosome; $\mathrm{R} / \mathrm{R}=$ relapsed/refractory.

${ }^{a}$ Minimal residual disease negativity defined according to molecular level $<0.01 \%$.

\section{Prophylaxis of CNS Leukemia}

Prophylactic CNS therapy in ALL is essential to prevent not only CNS, but also systemic relapse and includes intrethecal (MTX alone or with ARA-C and steroids), systemic HD MTX and ARA-C, and radiation therapy, the latter rarely used. With these modalities, the CNS relapse rate decreased to $\leq 5 \%$ and in trials with early HD-dose chemotherapy, CNS relapse rate was $\leq 2 \%$.

Effective CNS prophylaxis is even more crucial in patients with targeted therapies. In $\mathrm{Ph}^{+}$ALL, dasatinib and ponatinib cross the $\mathrm{BBB}$, whereas imatinib and nilotinib, as well as rituximab, inotuzumab, and blinatumomab do not.

Therapy of CNS Disease. At diagnosis, $5 \%$ to $10 \%$ of adult patients have CNS leukemia, with a prevalence in mature B-ALL and T-ALL (10\%-15\% and 10\%, respectively). In MLL-r cases, NG2 expression correlates with CNS involvement. For CNS leukemia at diagnosis, intrethecal chemotherapy is given 2 to 3 times/week, over 2 to 3 weeks until 2 consecutive cerebrospinal fluid (CSF) examinations are blast-free. If adult ALL patients with initial CNS involvement are treated adequately, they have no inferior outcome with regard to leukemia-free survival or CNS relapse rate and CNS involvement is no longer an adverse prognostic factor.

Relapse in the CNS is difficult to treat: in most cases, a bone marrow involvement is detected. Treatment consists of intrethecal chemotherapy, radiotherapy, and systemic therapy, the outcome is dismal and allo-SCT was the best option. CAR T-cells represent a promising option: a leptomeningeal infiltration was successfully treated with this approach and CAR T-cells detected in the CSF. ${ }^{76}$

\section{Immunotherapy}

Anti-CD19 Bi-Specific T-Cell Engager: Blinatumomab. CD19, virtually expressed on all B-ALL blasts, is an ideal target for antibody-directed therapy. Blinatumomab, the first bispecific T-cell engaging antibody construct, redirects host $\mathrm{CD}^{+} \mathrm{T}$ cells to $\mathrm{CD} 9^{+}$ALL cells. ${ }^{77}$

Minimal Residual Disease. Blinatumomab was first assessed in the MRD setting. Topp et al, used blinatumomab in 116 patients with ALL in first or later $\mathrm{MRD}^{+} \mathrm{CR}^{78}$ Seventy-eight patients achieved MRD negativity after 1 cycle and $80 \%$ after 4 . With a median followup of 29 months, the median survival was 36 months and median RFS months. ${ }^{75}$ Notably, allo-SCT did not confer a survival benefit.

Relapsed/Refractory ALL. In the confirmatory phase II study of 189 heavily pretreated patients with $\mathrm{R} / \mathrm{R} \mathrm{Ph}^{-}$ALL, blinatumomab was associated with a CR/CR with incomplete hematologic recovery rate of $43 \%$. The median response duration was 9 months and median survival 6 months. ${ }^{79}$ A phase III randomized trial (TOWER study) compared blinatumomab $(\mathrm{n}=271)$ with an investigator's choice chemotherapy (standard of care [SOC]; $\mathrm{n}=134$ ). The overall response rate was $45 \%$ with blinatumomab versus $30 \%$ with SOC, and CMR among responders $75 \%$ and $48 \%$, respectively. The

Table 6 Inotuzumab Activity

\begin{tabular}{|c|c|c|c|c|c|c|}
\hline Parameter & $\begin{array}{c}\text { Single Dose } \\
\text { Phase II }\end{array}$ & $\begin{array}{c}\text { Weekly Dose } \\
\text { Phase II }\end{array}$ & $\begin{array}{l}\text { Weekly Dose } \\
\text { Multicenter } \\
\text { Phase II }\end{array}$ & $\begin{array}{l}\text { INO-VATE } \\
\text { Phase III }\end{array}$ & $\begin{array}{l}\text { INO With Mini- } \\
\text { HCVD R/R ALL }\end{array}$ & $\begin{array}{l}\text { INO With Mini- } \\
\text { HCVD First-Line, } \\
\text { Elderly }\end{array}$ \\
\hline$n$ & 49 & 41 & 35 & 109 & 70 & 52 \\
\hline Dose/Schedule & $\begin{array}{l}1.8 \mathrm{mg} / \mathrm{m}^{2} \text { day } 1 \\
\text { every } 3-4 \text { weeks }\end{array}$ & $\begin{array}{c}0.8 \mathrm{mg} / \mathrm{m}^{2} \\
\text { day } 1,0.5 \mathrm{mg} / \mathrm{m}^{2} \\
\text { days } 8,15\end{array}$ & $\begin{array}{c}0.8 \mathrm{mg} / \mathrm{m}^{2} \\
\text { day } 1,0.5 \mathrm{mg} / \mathrm{m}^{2} \\
\text { days } 8,15\end{array}$ & $\begin{array}{c}0.8 \mathrm{mg} / \mathrm{m}^{2} \\
\text { day } 1,0.5 \mathrm{mg} / \mathrm{m}^{2} \\
\text { days } 8,15\end{array}$ & $\begin{array}{c}1.3-1.8 \mathrm{mg} / \mathrm{m}^{2} \text { in cycle } \\
1,1.0-1.3 \mathrm{mg} / \mathrm{m}^{2} \text { in } \\
\text { cycles } 2-4\end{array}$ & $\begin{array}{c}1.3-1.8 \mathrm{mg} / \mathrm{m}^{2} \text { in cycle } \\
1,1.0-1.3 \mathrm{mg} / \mathrm{m}^{2} \text { in } \\
\text { cycles } 2-4\end{array}$ \\
\hline \multicolumn{7}{|l|}{ Results } \\
\hline ORR (\%) & 57 & 59 & 68 & 88 & 77 & 97 \\
\hline CR (\%) & 18 & 20 & 31 & 36 & 59 & 80 \\
\hline $\begin{array}{l}\text { MRD negativity } \\
(\mathrm{FCM}, \%)\end{array}$ & 68 & 71 & 84 & 78 & 81 & 96 \\
\hline Median Survival, ms & 5 & 7.3 & 7.4 & 7.7 & 11 & Not reached \\
\hline
\end{tabular}

Abbreviations: $\mathrm{ALL}=$ acute lymphoblastic leukemia; FCM = flow cytometry; INO = inotuzumab ozogamicin; $\mathrm{MRD}=$ minimal residual disease; $\mathrm{R} / \mathrm{R}=\mathrm{relapsed} / \mathrm{refractory}$. 


\section{Treatment of ALL}

median survival was 7.7 and 4.0 months with blinatumomab versus SOC, respectively, with better results in salvage 1 patients (Table 5). ${ }^{80}$

Toxicity consists of fever, and chills—due to a cytokine release syndrome (CRS), and hypogammaglobulinemia. Tremor, headache, other mental status changes, and rarely seizures were reported. Serious adverse events are uncommon, and include encephalopathy.

\section{Inotuzumab Ozogamicin}

CD22 is expressed in 95\% B-ALL and universally in Burkitt leukemia. Inotuzumab ozogamicin comprises an anti-CD22 antibody linked to calicheamicin. ${ }^{81}$ In a single institution phase II study including 49 patients with R/R ALL, inotuzumab was administered at a starting dose of 1.3 to $1.8 \mathrm{mg} / \mathrm{m}^{2}$ intravenously every 3 to 4 weeks. ${ }^{82}$ The objective response rate was $57 \%$, and median survival 5.1 months; nearly half of the patients treated proceeded to alloSCT. Common adverse effects included fever and hypotension. Serious toxicities included veno-occlusive disease (VOD) post alloSCT (23\%), mainly observed in older patients receiving double alkylators during conditioning. To minimize toxicities, inotuzumab was administered on a different schedule in $40 \mathrm{R} / \mathrm{R}$ ALL patients, ${ }^{83}$ resulting in similar responses (59\%), fewer adverse events, and lower rates of VOD. Similar results were reported in a multicenter phase II trial. ${ }^{84}$

Finally, in a randomized phase III trial comparing inotuzumab with SOC in R/R ALL (salvage 1 and 2), objective response rates were $88 \%$ (CR 81\%) with inotuzumab and 32\% (CR 29\%) with SOC. Among responders, the MRD-negativity rates were $78 \%$ and $28 \%$, respectively. The median PFS was 5.0 with inotuzumab versus 1.8 months with SOC, median survival was 7.7 versus 6.7 months and 2 -year survival was $23 \%$ versus $10 \%{ }^{85}$

\section{Combination Therapy in R/R ALL}

Inotuzumab was evaluated in the $\mathrm{R} / \mathrm{R}$ setting in combination "mini-hyper-ìcyclophosphamide, vincristine and dexamethasone (CVD)": 59 patients were treated, leading to objective response in $78 \%$ (CR 59\%), with $82 \%$ of responders achieving MRD negativity. The 2-year PFS and OS rates were $60 \%$ and $32 \%$, respectively; better results were observed in salvage 1 patients. The survival of patients treated with mini-hyper-CVD with inotuzumab were superior to a historical cohort treated with inotuzumab monotherapy (median survival, 11 months vs. 6 months). ${ }^{86}$ Studies exploring lower doses of inotuzumab are ongoing (Table 6).

\section{Other Agents in Development}

Other antibody drug conjugates (ADC) targeting CD19 and $\mathrm{CD} 22$ are in development. Among them, anti-CD19 PBD-conjugate (ADCT-402) is an ADC composed of a humanized monoclonal antibody directed against human CD19, conjugated to SG3199, a pyrrolobenzodiazepine dimer cytotoxin. ${ }^{87}$ In a phase I study in $29 \mathrm{R} /$ $\mathrm{R}$ ALL patients, ADCT-402 was well tolerated. Four patients responded at the higher-dose levels (3 CR, 1 complete remission with incomplete recovery [CRi]), 2 achieving MRD negativity.

\section{Chimeric Antigen Receptor T-Cell Therapies}

Chimeric antigen receptor T-cells are a recent development in cancer treatment. ${ }^{88}$ CAR T cells directed at CD19 are effective for patients with aggressive B-cell lymphomas and pediatric ALL. In the initial study, 59 children with R/R ALL were treated. ${ }^{89}$ The CR rate was $93 \%$. The estimated 1 -year EFS and OS were $55 \%$ and $79 \%$, respectively. CRS occurred in $88 \%$ of patients, all of whom recovered. In a confirmatory phase I/II, 25-center, global study, 75 patients aged 3 to 23 years (median age, 11 years) were treated. ${ }^{90}$ The overall remission rate within 3 months was 59\% (83\% among patients who were evaluable for efficacy). All responders achieved negative MRD status. The EFS and OS rates were $50 \%$ and $76 \%$ at 12 months, respectively.

In the ZUMA-3 study, 33 patients with R/R ALL were treated. The overall response rate was $71 \%$ (CR 67\% and CRi 4\%). ${ }^{91}$ Overall, the rate of Grade $\geq 3$ CRS was $28 \%$; and any Grade $\geq 3$ neurologic events was 52\%.

Recently, an adult study of CD19 CAR T-cells was reported. ${ }^{92}$ Eighty-three patients were enrolled, 78 underwent apheresis and 53 were treated. CR was observed in 44 of 53 patients treated (83\%; $\mathrm{CR}$ in 44 of 78 who underwent apheresis $=56 \%$ ). Median EFS was 6.1 months and median survival was 12.9 months for the patients treated. The 2-year EFS and survival rates were approximately $15 \%$ and $<30 \%$, respectively. Patients with marrow blasts $<5 \%$ had longer EFS and survival durations, and lower incidences of CRS and neurotoxic events.

To circumvent CD19 escape after CD19-CAR T-cell therapy, CD22-targeted CAR T-cell therapy has recently been developed. ${ }^{93}$ Of the 15 children and adults with R/R B-ALL, most who were previously treated with CD-19 directed immunotherapy, 11 (73\%) achieved CR after treatment with $\geq 1 \times 10^{6} / \mathrm{kg}$. Current CAR T-cell therapies use autologous lymphocytes, which can be troublesome: new platforms provide an "off-the-shelf" approach, derived from healthy volunteer donors. Preliminary results of the CALM study using this therapy in a phase I dose-escalation trial showed that in 6 adults, 4 achieved CRi with MRD negativity. ${ }^{93}$ Off-theshelf products targeting CD22 and allogeneic cord blood-derived natural killer cells are being developed.

\section{Concluding Key Points}

- The treatment of ALL still relies on the use of chemotherapy, with survival rates ranging from $50 \%$ to $70 \%$ in AYA.

- Risk stratification relies on MRD, and must be integrated with the genetic profile.

- Targeted treatment (TKI, and immunotherapy) are opening a new era in ALL.

- Philadelphia chromosome-positive ALL long-term outcome improved from $10 \%$ to $60 \%$ in recent years, and is likely to improve even further

- Allogeneic SCT recommendation must be reconsidered in light of all these key points.

\section{Acknowledgments}

The authors thank Associazione Italiana per la Ricerca sul Cancro, Special Program Molecular Clinical Oncology-Extension program, $5 \times 1000$ (10007), Milan (Italy) to Robin Foà; and Finanziamento Medi Progetti Universitari 2015 to S.C. (Sapienza University of Rome). 


\section{Disclosure}

Dr Chiaretti received fees as a speaker from received grants: Amgen; Incyte, Shire, Pfizer; Dr Jabbour has received research grants from Pfizer, Amgen, Abbvie, Takeda, and Novartis; and Dr Hoelzer has an ownership interest in Amgen, Shire, Medac, Jazz Pharma, Erytech, Celgene, Juno, Erytech, and DSMC.

\section{References}

1. Kalina T, Flores-Montero J, van der Velden VH, et al. EuroFlow standardization of flow cytometer instrument settings and immunophenotyping protocols. Leukemia 2012; 26:1986-2010.

2. van Dongen JJ, Lhermitte L, Böttcher S, et al. EuroFlow antibody panels for standardized n-dimensional flow cytometric immunophenotyping of normal, reactive and malignant leukocytes. Leukemia 2012; 26:1908-75.

3. Vitale A, Guarini A, Ariola C, et al. Absence of prognostic impact of CD13 and/or CD33 antigen expression in adult acute lymphoblastic leukemia. Results of the GIMEMA ALL 0496 trial. Haematologica 2007; 92:342-8.

4. Chiaretti S, Vitale A, Cazzaniga G, et al. Clinico-biological features of 5202 patients with acute lymphoblastic leukemia enrolled in the Italian AIEOP and GIMEMA protocols and stratified in age cohorts. Haematologica 2013; 98:1702-10.

5. Zangrando A, Intini F, te Kronnie G, Basso G. Validation of NG2 antigen in identifying BP-ALL patients with MLL rearrangements using qualitative and quantitative flow cytometry: a prospective study. Leukemia 2008; 22:858-61.

6. Tang GS, Wu J, Liu M, et al. BCR-ABL1 and CD66c exhibit high concordance in minimal residual disease detection of adult B-acute lymphoblastic leukemia. Am J Transl Res 2015; 7:632-9.

7. Arber DA, Orazi A, Hasserjian R, et al. The 2016 revision to the World Health Organization classification of myeloid neoplasms and acute leukemia. Blood 2016; 127:2391-405.

8. Coustan-Smith E, Mullighan CG, Onciu M, et al. Early T-cell precursor leukaemia: a subtype of very high-risk acute lymphoblastic leukaemia. Lancet Oncol 2009; 10:147-56.

9. Burmeister T, Gökbuget N, Schwartz S, et al. Clinical features and prognostic implications of TCF3-PBX1 and ETV6-RUNX1 in adult acute lymphoblastic leukemia. Haematologica 2010; 95:241-6.

10. Moorman AV, Robinson H, Schwab C, et al. Risk-directed treatment intensification significantly reduces the risk of relapse among children and adolescents with acute lymphoblastic leukemia and intrachromosomal amplification of chromosome 21: a comparison of the MRC ALL97/99 and UKALL2003 trials. J Clin Oncol 2013; 31:3389-96.

11. Russell LJ, Enshaei A, Jones L, et al. IGH@ translocations are prevalent in teenagers and young adults with acute lymphoblastic leukemia and are associated with a poor outcome. J Clin Oncol 2014; 32:1453-62.

12. Fischer U, Forster M, Rinaldi A, et al. Genomics and drug profiling of fatal TCF3HLF-positive acute lymphoblastic leukemia identifies recurrent mutation patterns and therapeutic options. Nat Genet 2015; 47:1020-9.

13. Chiaretti S, Foa R. Management of adult Ph-positive acute lymphoblastic leukemia. Hematol Am Soc Hematol Educ Program 2015; 2015:406-13.

14. Schneider NR, Carroll AJ, Shuster JJ, et al. New recurring cytogenetic abnormalities and association of blast cell karyotypes with prognosis in childhood T-cell acute lymphoblastic leukemia: a pediatric oncology group report of 343 cases. Blood 2000; 96:2543-9.

15. Lange BJ, Raimondi SC, Heerema N, et al. Pediatric leukemia/lymphoma with t(8;14)(q24;q11). Leukemia 1992; 6:613-8.

16. Mullighan CG, Goorha S, Radtke I, et al. Genome-wide analysis of genetic alterations in acute lymphoblastic leukaemia. Nature 2007; 446:758-64.

17. Mullighan CG, Su X, Zhang J, et al. Deletion of IKZF1 and prognosis in acute lymphoblastic leukemia. N Engl J Med 2009; 360:470-80.

18. Martinelli G, Iacobucci I, Storlazzi CT, et al. IKZF1 (Ikaros) deletions in BCRABL1-positive acute lymphoblastic leukemia are associated with short disease-free survival and high rate of cumulative incidence of relapse: a GIMEMA AL WP report. J Clin Oncol 2009; 27:5202-7.

19. Ribera J, Morgades M, Zamora L, et al. Prognostic significance of copy number alterations in adolescent and adult patients with precursor B acute lymphoblastic leukemia enrolled in PETHEMA protocols. Cancer 2015; 121:3809-17.

20. Messina M, Chiaretti S, Fedullo AL, et al. Clinical significance of recurrent copy number aberrations in B-lineage acute lymphoblastic leukaemia without recurrent fusion genes across age cohorts. Br J Haematol 2017; 178:583-7.

21. Mullighan CG. Molecular genetics of B-precursor acute lymphoblastic leukemia. J Clin Invest 2012; 122:3407-15.

22. Roberts KG, Li Y, Payne-Turner D, et al. Targetable kinase-activating lesions in Ph-like acute lymphoblastic leukemia. N Engl J Med 2014; 371:1005-15.

23. Zhang J, McCastlain K, Yoshihara H, et al. Deregulation of DUX4 and ERG in acute lymphoblastic leukemia. Nat Genet 2016; 48:1481-9.

24. Chiaretti S, Gianfelici V, O’Brien SM, Mullighan CG. Advances in the genetics and therapy of acute lymphoblastic leukemia. Am Soc Clin Oncol Educ Book 2016 35:e314-22.
25. Hoelzer D, Bassan R, Dombret H, et al. Acute lymphoblastic leukaemia in adult patients: ESMO Clinical Practice Guidelines for diagnosis, treatment and followup. Ann Oncol 2016; 27:v69-82.

26. van Dongen JJ, van der Velden VH, Brüggemann M, Orfao A. Minimal residual disease diagnostics in acute lymphoblastic leukemia: need for sensitive, fast, and standardized technologies. Blood 2015; 125:3996-4009.

27. Ladetto M, Brüggemann M, Monitillo L, et al. Next-generation sequencing and real-time quantitative PCR for minimal residual disease detection in B-cell disorders. Leukemia 2014; 28:1299-307.

28. Della Starza I, Nunes V, Cavalli M, et al. Comparative analysis between RQ-PCR and digital-droplet-PCR of immunoglobulin/T-cell receptor gene rearrangements to monitor minimal residual disease in acute lymphoblastic leukaemia. $\mathrm{Br} J$ Haematol 2016; 174:541-9.

29. Hoelzer D. Clinical applications and pitfalls of MRD in ALL. Clin Lymphoma Myeloma Leuk 2017; 17:S9-11.

30. Berry DA, Zhou S, Higley H, et al. Association of minimal residual disease with clinical outcome in pediatric and adult acute lymphoblastic leukemia: a metaanalysis. JAMA Oncol 2017; 3:e170580.

31. Gökbuget N, Brüggemann M, Beck J, et al. Evaluation of minimal residual disease (MRD) and MRD-based treatment decisions in Ph/BCR-ABL negative adult acute lymphoblastic leukemia (ALL): experience from the German Multicenter Study Group for Adult ALL (GMALL) (abstract 2017). Blood 2017:130.

32. Kantarjian H, Thomas D, O'Brien S, et al. Long-term follow-up results of hyperfractionated cyclophosphamide, vincristine, doxorubicin, and dexamethasone (Hyper-CVAD), a dose-intensive regimen, in adult acute lymphocytic leukemia. Cancer 2004; 101:2788-801.

33. Annino L, Vegna L, Camera A, et al. Treatment of adult acute lymphoblastic leukemia (ALL): long-term follow-up of the GIMEMA ALL 0288 randomized study. Blood 2002; 99:863-71.

34. Marks DI, Paietta EM, Moorman AV, et al. T-cell acute lymphoblastic leukemia in adults: clinical features, immunophenotype, cytogenetics, and outcome from the large randomized prospective trial (UKALL XII/ECOG 2993). Blood 2009; 114 : 5136-45.

35. Hoelzer D, Walewski J, Döhner H, et al. Improved outcome of adult Burkitt lymphoma/leukemia with rituximab and chemotherapy: report of a large prospective multicenter trial. Blood 2014; 124:3870-9.

36. Ram R, Wolach O, Vidal L, et al. Adolescents and young adults with acute lymphoblastic leukemia have a better outcome when treated with pediatric-inspired regimens: systematic review and meta-analysis. Am J Hematol 2012; 87:472-8.

37. Ribera JM, Ribera J, Genescà E. Treatment of adolescent and young adults with acute lymphoblastic leukemia. Mediterr J Hematol Infect Dis 2012; 6:e2014052.

38. Burke PW, Douer D. Acute lymphoblastic leukemia in adolescents and young adults. Acta Haematol 2014; 132:264-73.

39. McNeer JL, Raetz EA. Acute lymphoblastic leukemia in young adults: which treatment? Curr Opin Oncol 2012; 24:487-94.

40. Rytting ME, Thomas DA, O'Brien S, et al. Augmented Berlin-Frankfurt-Münster therapy in adolescents and young adults (AYAs) with acute lymphoblastic leukemia (ALL). Cancer 2014; 120:3660-8.

41. Gökbuget N, Beck J, Brandt K, et al. Significant improvement of outcome in adolescents and young adults (AYAs) aged 15-35 years with acute lymphoblastic leukemia (ALL) with a pediatric derived adult ALL protocol; results of 1529 AYAs in 2 consecutive trials of the German Multicenter Study Group For Adult ALL (GMALL) (abstract 839). Blood 2013:122.

42. Huguet F, Leguay T, Raffoux E, et al. Pediatric-inspired therapy in adults with Philadelphia chromosome-negative acute lymphoblastic leukemia: the GRAALL2003 study. J Clin Oncol 2009; 27:911-8.

43. DeAngelo DJ, Stevenson KE, Dahlberg SE, et al. Long-term outcome of a pediatric-inspired regimen used for adults aged $18-50$ years with newly diagnosed acute lymphoblastic leukemia. Lenkemia 2015; 29:526-34.

44. Guru Murthy GS, Venkitachalam R, Mehta P. Trends in survival outcomes of Blineage acute lymphoblastic leukemia in elderly patients: analysis of Surveillance, Epidemiology, and End Results database. Leuk Lymphoma 2015; 56:2296-300.

45. Gökbuget N. How I treat older patients with ALL. Blood 2013; 122:1366-75.

46. Geyer MB, Hsu M, Devlin SM, Tallman MS, Douer D, Park JH. Overall survival among older US adults with ALL remains low despite modest improvement since 1980: SEER analysis. Blood 2017; 129:1878-81.

47. Kantarjian H, Ravandi F, Short NJ, et al. Inotuzumab ozogamicin in combination with low-intensity chemotherapy for older patients with Philadelphia chromosome-negative acute lymphoblastic leukaemia: a single-arm, phase 2 study. Lancet Oncol 2018; 19:240-8.

48. Rousselot P, Coudé MM, Gokbuget N, et al. Dasatinib and low-intensity chemotherapy in elderly patients with Philadelphia chromosome-positive ALL. Blood 2016; 128:774-82

49. Foà R, Vitale A, Vignetti M, et al. Dasatinib as first-line treatment for adult patients with Philadelphia chromosome-positive acute lymphoblastic leukemia. Blood 2011; 118:6521-8.

50. Ottmann OG, Pfeifer H, Cayuela JM, et al. Nilotinib (Tasigna) and chemotherapy for first-line treatment in elderly patients with De Novo Philadelphia chromosome/ BCR-ABL1 positive acute lymphoblastic leukemia (ALL): A Trial of the European Working Group for Adult ALL (EWALL-PH-02) (abstract 798). Blood 2014:124.

51. Short N, Kantarjian K, Ravandi F, et al. Frontline hyper-CVAD plus ponatinib for patients with Philadelphia chromosome-positive acute lymphoblastic leukemia: 


\section{Treatment of ALL}

updated results of a phase II study (abstract 7013). J Clin Oncol 2017; 35(15 suppl).

52. Martinelli G, Piciocchi A, Papayannidis C, et al. First report of the Gimema LAL1811 phase II prospective study of the combination of steroids with ponatinib as frontline therapy of elderly or unfit patients with Philadelphia chromosomepositive acute lymphoblastic leukemia (abstract 99). Blood 2017:130.

53. Chalandon Y, Thomas X, Hayette S, et al. Randomized study of reduced-intensity chemotherapy combined with imatinib in adults with Ph-positive acute lymphoblastic leukemia. Blood 2015; 125:3711-9.

54. Giebel S, Labopin M, Gorin NC, et al. Improving results of autologous stem cel transplantation for Philadelphia-positive acute lymphoblastic leukaemia in the era of tyrosine kinase inhibitors: a report from the Acute Leukaemia Working Party of the European Group for Blood and Marrow Transplantation. Eur J Cancer 2014; 50:411-7.

55. Pfeifer H, Wassmann B, Bethge W, et al. Randomized comparison of prophylactic and minimal residual disease-triggered imatinib after allogeneic stem cell transplantation for BCR-ABL1-positive acute lymphoblastic leukemia. Leukemia 2013; 27:1254-62.

56. Carpenter PA, Johnston L, Fernandez HF, et al. Posttransplant feasibility study of nilotinib prophylaxis for high-risk Philadelphia chromosome positive leukemia. Blood 2017; 130:1170-2.

57. Ravandi F, Othus M, O'Brien S, et al. US intergroup study of chemotherapy plus dasatinib and allogeneic stem cell transplant in Philadelphia chromosome positive ALL. Blood Adv 2016; 1:250-9.

58. Zabriskie MS, Eide CA, Tantravahi SK, et al. BCR/ABL1 compound mutations combining key kinase domain positions confer clinical resistance to ponatinib in $\mathrm{Ph}$ chromosome-positive leukemia. Cancer Cell 2014; 26:428-42.

59. Pemovska T, Johnson E, Kontro M, et al. Axitinib effectively inhibits BCRABL1(T315I) with a distinct binding conformation. Nature 2015; 519:102-5.

60. Wylie AA, Schoepfer J, Jahnke W, et al. The allosteric inhibitor ABL001 enables dual targeting of BCR-ABL1. Nature 2017; 543:733-7.

61. Martinelli G, Boissel N, Chevallier P, et al. Complete hematologic and molecular response in adult patients with relapsed/refractory Philadelphia chromosome-positive B-precursor acute lymphoblastic leukemia following treatment with blinatumomab: results from a phase II, single-arm, multicenter study. J Clin Oncol 2017; 35:1795-802.

62. Hanif A, Wang ES, Thompson JE, et al. Combining blinatumomab with targeted therapy for BCR-ABL mutant relapsed/refractory acute lymphoblastic leukemia. Leuk Lymphoma 2018; 18:1-3.

63. Assi R, Kantarjian H, Short NJ, et al. Safety and efficacy of blinatumomab in combination with a tyrosine kinase inhibitor for the treatment of relapsed Philadelphia chromosome-positive leukemia. Clin Lymphoma Myeloma Leuk 2017; 17: 897-901.

64. Jain N, Cortes JE, Ravandi F, et al. Inotuzumab ozogamicin in combination with bosutinib for patients with relapsed or refractory $\mathrm{Ph}^{+} \mathrm{ALL}$ or CML in lymphoid blast phase (abstract 143). Blood 2017:130.

65. Roberts KG, Pei D, Campana D, et al. Outcomes of children with BCR-ABL1-like acute lymphoblastic leukemia treated with risk-directed therapy based on the levels of minimal residual disease. J Clin Oncol 2014; 32:312-20.

66. Roberts KG, Gu Z, Payne-Turner D, et al. High frequency and poor outcome of Philadelphia chromosome-like acute lymphoblastic leukemia in adults. I Clin Oncol 2017; 35:394-401.

67. Jain N, Jabbour EJ, McKay PZ, et al. Ruxolitinib or dasatinib in combination with chemotherapy for patients with relapsed/refractory Philadelphia ( $\mathrm{Ph}$ )-like acute lymphoblastic leukemia: a phase I-II trial (abstract 1322). Blood 2017; 130.

68. Chiaretti S, Messina M, Grammatico S, et al. Rapid identification of $B C R / A B L 1$ like acute lymphoblastic leukemia patients using a predictive statistical model based on quantitative real time-polymerase chain reaction: clinical, prognostic and therapeutic implications. Br J Haematol, in press.

69. Neumann M, Coskun E, Fransecky L, et al. FLT3 mutations in early T-cel precursor ALL characterize a stem cell like leukemia and imply the clinical use of tyrosine kinase inhibitors. PLoS One 2013; 8:e53190.

70. Bond J, Marchand T, Touzart A, et al. An early thymic precursor phenotype predicts outcome exclusively in HOXA-overexpressing adult T-cell acute lymphoblastic leukemia: a Group for Research in Adult Acute Lymphoblastic Leukemia study. Haematologica 2016; 101:732-40.

71. Bond J, Graux C, Lhermitte L, et al. Early response-based therapy stratification improves survival in adult early thymic precursor acute lymphoblastic leukemia: a
Group for Research on Adult Acute Lymphoblastic Leukemia Study. J Clin Oncol 2017; 35:2683-91.

72. Peirs S, Frismantas V, Matthijssens F, et al. Targeting BET proteins improves the therapeutic efficacy of BCL-2 inhibition in T-cell acute lymphoblastic leukemia. Leukemia 2017; 31:2037-47.

73. Gökbuget N, Kneba M, Raff T, et al. Adult patients with acute lymphoblastic leukemia and molecular failure display a poor prognosis and are candidates for stem cell transplantation and targeted therapies. Blood 2012; 120:1868-76.

74. Bassan R, Spinelli O, Oldani E, et al. Improved risk classification for risk-specific therapy based on the molecular study of minimal residual disease (MRD) in adult acute lymphoblastic leukemia (ALL). Blood 2009; 113:4153-62.

75. Gökbuget N, Dombret H, Bonifacio $\mathrm{M}$, et al. Blinatumomab for minimal residual disease in adults with B-precursor acute lymphoblastic leukemia. Blood 2018. https://doi.org/10.1182/blood-2017-08-798322, Published online January 22.

76. Lee DW, Kochenderfer JN, Stetler-Stevenson M, et al. T cells expressing CD19 chimeric antigen receptors for acute lymphoblastic leukaemia in children and young adults: a phase 1 dose-escalation trial. Lancet 2015; 385:517-28.

77. Nagorsen D, Kufer P, Baeuerle PA, Bargou R. Blinatumomab: a historical perspective. Pharmacol Ther 2012; 136:334-42.

78. Topp MS, Kufer P, Gökbuget N, et al. Targeted therapy with the T-cell-engaging antibody blinatumomab of chemotherapy-refractory minimal residual disease in B-lineage acute lymphoblastic leukemia patients results in high response rate and prolonged leukemia-free survival. J Clin Oncol 2011; 29:2493-8.

79. Topp MS, Gökbuget N, Stein AS, et al. Safety and activity of blinatumomab for adult patients with relapsed or refractory B-precursor acute lymphoblastic leukaemia: a multicentre, single-arm, phase 2 study. Lancet Oncol 2015; 16:57-66.

80. Kantarjian H, Stein A, Gökbuget N, et al. Blinatumomab versus chemotherapy for advanced acute lymphoblastic leukemia. N Engl J Med 2017; 376:836-47.

81. Thomas X. Inotuzumab ozogamic in in the treatment of B-cell acute lymphoblastic leukemia. Expert Opin Investig Drugs 2012; 21:871-8.

82. Kantarjian H, Thomas D, Jorgensen J, et al. Inotuzumab ozogamicin, an antiCD22-calicheamicin conjugate, for refractory and relapsed acute lymphocytic leukaemia: a phase 2 study. Lancet Oncol 2012; 13:403-11.

83. Kantarjian H, Thomas D, Jorgensen J, et al. Results of inotuzumab ozogamicin, a CD22 monoclonal antibody, in refractory and relapsed acute lymphocytic leukemia. Cancer 2013; 119:2728-36.

84. DeAngelo DJ, Stock W, Stein AS, et al. Inotuzumab ozogamicin in adults with relapsed or refractory CD22-positive acute lymphoblastic leukemia: a phase 1/2 study. Blood Adv 2017; 1:1167-80.

85. Kantarjian HM, DeAngelo DJ, Stelljes M, et al. Inotuzumab ozogamicin versus standard therapy for acute lymphoblastic leukemia. N Engl J Med 2016; 375:740-53.

86. Jabbour E, Ravandi F, Kebriaei P, et al. Salvage chemoimmunotherapy with inotuzumab ozogamicin combined with mini-hyper-CVD for patients with relapsed/refractory Philadelphia chromosome-negative acute lymphoblastic leukemia. JAMA Oncol 2018; 4:230-4.

87. Jain N, Klisovic RB, Stock W, et al. Interim data from a phase 1 study evaluating pyrrolobenzodiazepine-based antibody drug conjugate ADCT-402 (loncastuximab tesirine) targeting CD19 for relapsed or refractory B-cell acute lymphoblastic leukemia (abstract 1321). Blood 2017; 130.

88. Hartmann J, Schüßler-Lenz M, Bondanza A, Buchholz CJ. Clinical development of CAR T cells - challenges and opportunities in translating innovative treatment concepts. EMBO Mol Med 2017; 9:1183-97.

89. Brentjens RJ, Davila ML, Riviere I, et al. CD19-targeted T cells rapidly induce molecular remissions in adults with chemotherapy refractory acute lymphoblastic leukemia. Sci Transl Med 2013; 5:177ra38.

90. Grupp SA, Laetsch TW, Buechner J, et al. Analysis of a global registration trial of the efficacy and safety of CTL019 in pediatric and young adults with relapsed/ refractory acute lymphoblastic leukemia (ALL) (abstract 221). Blood 2016; 128.

91. Shah BD, Stock W, Wierda WG, et al. Phase 1 results of ZUMA-3: KTE-C19, an antiCD19 chimeric antigen receptor (CAR) T cell therapy, in adult patients with relapsed/ refractory acute lymphoblastic leukemia (R/R ALL) (abstract 888). Blood 2017:130.

92. Fry TJ, Shah NN, Orentas RJ, et al. CD22-targeted CAR T cells induce remission in B-ALL that is naive or resistant to CD19-targeted CAR immunotherapy. Nat Med 2018; 24:20-8.

93. Graham C, Yallop D, Jozwik A, et al. Preliminary results of UCART19, an allogeneic anti-CD19 CAR T-cell product, in a first-in-human trial (CALM) in adult patients with CD19+ relapsed/refractory B-cell acute lymphoblastic leukemia (abstract 887). Blood 2017:130. 